\title{
The effect of normotensive arterial pressure on cerebral saturation during carotid endarterectomy
}

\author{
(DCihan Yücel, @Nihan Kayalar \\ Prof. Dr. Cemil Taşcıoğlu City Hospital, Department of Cardiovascular Surgery, İstanbul, Turkey
}

Cite this article as: Yücel C, Kayalar N. The effect of normotensive arterial pressure on cerebral saturation during carotid endarterectomy. J Health Sci Med 2021; 4(2): 181-185.

\begin{abstract}
Aim: Monitoring of cerebral perfusion during carotid endarterectomy helps to institute early intervention to prevent risk of ischemia during the operation. We aimed to evaluate if the disadvantages of high systemic pressure can be avoided by keeping arterial blood pressure below $150 \mathrm{mmHg}$ during cross-clamping of the internal carotid artery by correlating our findings of NIRS values with clinical results.

Material and Method: This was designed as a prospective study that included elective patients scheduled for an operation of carotid artery stenosis under general anesthesia. A total of 60 patients operated on between August and October 2019 were included in the study.

Results: Patients' NIRS findings on the right and left hemispheres were analyzed separately to be able to differentiate any possible changes of NIRS of the cerebral hemisphere ipsilateral to the clamped carotid artery. The analysis of the right and left NIRS findings in these 60 patients revealed a significant decrease between intubation and incision NIRS values ( $\mathrm{p}=0.008$ and $\mathrm{p}=0.02$ respectively).

Conclusion: Considering the NIRS findings and clinical observations of the patients in the postoperative period, we think that ICA endarterectomy operations may be safe to perform in a suitable patient group while the systolic arterial blood pressure is maintained below $150 \mathrm{mmHg}$ during cross-clamping.
\end{abstract}

Keywords: Carotid endarterectomy, near-infrared spectroscopy, stroke

\section{INTRODUCTION}

The technique of carotid endarterectomy is known to bear a relatively low perioperative stroke risk of around 0.7 $1.5 \%$. However, it remains to be a social health problem in terms of outcomes and long duration of treatment after stroke despite this relatively low risk (1). Monitoring of cerebral perfusion during carotid endarterectomy (CEA) helps to institute early intervention to prevent risk of ischemia during the operation.

The awake test under regional anesthesia is the most reliable method among all techniques of cerebral perfusion monitoring (2). However, different methods are used to monitor cerebral perfusion such as Transcranial doppler (TCD) and Near-Infrared Spectroscopy (NIRS) which have come into prominence in recent years (3). NIRS is a test that can measure changes in cerebral oxygenation continuously and non-invasively. NIRS measures regional oxygenation by interpreting oxyhemoglobin and deoxyhemoglobin signals. Normal cerebral NIRS values are around $60 \%$ (4). A decrease in regional cerebral oxygen saturation $\left(\mathrm{rScO}_{2}\right)$ below $40 \%$ or $25 \%$ decline from baseline may be indicative of cerebral ischemia (5).

The development of cerebral ischemia during carotid endarterectomy usually requires insertion of a shunt to prevent stroke. Another time honoured approach is to increase blood pressure during carotid clamping although there are no studies showing the effects of blood pressure changes on NIRS values at the time of clamping. There are also no studies related to the clinical results of blood pressure management strategies during carotid surgery. In our study, we examined patients with carotid artery stenosis operated on under general anesthesia. We aimed to evaluate if the disadvantages of high systemic pressure can be avoided by keeping arterial blood pressure below $150 \mathrm{mmHg}$ during cross-clamping of the internal carotid artery by correlating our findings of NIRS values and clinical results. 


\section{MATERIAL AND METHOD}

This was designed as a prospective study that included elective patients scheduled for an operation of carotid artery stenosis undergeneral anesthesia. Ethical approval and informed consent of the patients were obtained. The study was carried out with the permission of Ethics Committee of Okmeydanı Training and Research Hospital (ethic number: 48670771-514.10). A total of 60 patients operated on between August and October 2019 were included in the study. All patients underwent carotid CT angiography before the operation. Patients with unilateral or bilateral carotid artery stenosis above $70 \%$ were operated (6). All patients underwent a detailed preoperative cardiac evaluation.

Invasive radial arterial pressure monitoring was performed along with standard monitorization. The frontal region was cleansed before the patients were anesthetized and NIRS probes were placed on the left and right sides of the frontal region over the eyebrows. Preanaesthetic NIRS values of the right and left sides, systolic and diastolic arterial pressures, mean arterial pressure (MAP), peripheral oxygen saturation $\left(\mathrm{SpO}_{2}\right)$ were recorded. These values were continuously monitored and recorded at intubation, at incision, and every 5 minutes starting from the first minute of ICA cross clamping. The values after removal of carotid clamp, at the end of operation and after the extubation of patients were also recorded.

A standard longitudinal endarterectomy was performed in all patients with primary or patch closure of arteriotomy with ven graft, depending on the arterial diameter and surgeon preference. Our strategy to use shunting is when NIRS values drop $20-25 \%$ from baseline or when the clamp time is anticipated to be long due to the anatomy of the patient and the carotid lesion. 5000 units of systemic intravenous (IV) heparin were administered before the carotid arterial cross-clamp was placed with the aim of an ACT value above 200 . The blood pressure was controlled in order to prevent its increase and systolic pressure was kept below 150 mmHg. Cross-clamp durations were recorded.

Patients were taken to the Cardiovascular Surgery Intensive Care Unit after the operation. Patients were followed up closely for hemodynamic and neurological sequelae. Major cerebral event was defined as cerebral infarction or cerebral hemorrhage within postoperative 30 days, while minor events were defined as transient hemiplegia or hemiparesis with no pathology detected on MR/CT.

\section{Statistical Analysis}

The data obtained from the study were analyzed using the SPSS (Statistical Package for Social Sciences) version
21 package program. In the descriptive statistics, categorical variables were expressed as numbers and percentages, while numerical variables were expressed as mean, standard deviation, median, minimum and maximum values, and interquartile range (IQR). The Shapiro Wilk test was used to evaluate the normality of variable distribution. In the comparison of two independent groups, the Student T-test was used for numerical variables with normal distribution while the non-parametric Mann Whitney $U$ test was used when the assumptions for a parametric test were not met. The repeated measures ANOVA was used to evaluate repeated NIRS values during and after the operation. The Mauchly's test was used to test for sphericity and the Greenhouse-Geisser correction was used when the assumption of sphericity was violated. The correlational analysis was performed by Pearson's tests for parametric conditions while the Spearman's tests were used for non-parametric conditions. The type- 1 error rate below $5 \%(\mathrm{p}<0.05)$ was considered statistically significant.

\section{RESULTS}

Between August 2019 and October 2019, carotid endarterectomy was performed in a total of 60 patients (16 women (26.7\%), 44 men (73.3\%) with a mean age of $68.27 \pm 6.62$ years. All patient data such as demographics, smoking status, concomitant diseases, neurological symptoms (previous stroke, transient ischemic attack (TIA), ejection fraction (EF), and the presence of bilateral carotid artery lesions were recorded. Bilateral lesion was defined as carotid stenosis $>70 \%$ in both carotid arteries.

Hypertension was the most common concomitant disease among patients (46 patients (76.7\%). Of the patients, $32(53.3 \%)$ were asymptomatic. All our symptomatic patients were recently referred with new onset of symptoms which started within a few months. The mean ejection fraction (EF) was $55.17 \pm 5.49 \%$. Patients' clinical characteristics and comorbidities are given in Table 1.

A total of 14 patients $(23.3 \%)$ had bilateral carotid artery disease. 22 patients (36.7\%) underwent right carotid endarterectomy and 38 (63.3\%) underwent left carotid endarterectomy. Saphenous patch plasty was performed in 10 patients $(16.6 \%)$. No postoperative mortality, myocardial infarction or permanent stroke was recorded, but one patient (1.6\%) had a transient ischemic attack. Patient's MRI showed no lesions and it was thought to result from hypoperfusion although intraoperative NIRS values did not show a significant decrease. Patients' operative and postoperative characteristics are given in Table 2. 


\begin{tabular}{|c|c|c|}
\hline Age (years) & $68.27 \pm 6.6$ & $54-82$ \\
\hline Ejection fraction & $55.17 \pm 5.49$ & 45-65 \\
\hline Variable & $\mathbf{n}$ & $\%$ of total \\
\hline \multicolumn{3}{|l|}{ Sex } \\
\hline Male & 44 & 73.3 \\
\hline Female & 16 & 26.7 \\
\hline Hypertension & 46 & 76.7 \\
\hline Smoking & 34 & 56.7 \\
\hline Diabetes mellitus & 32 & 53.3 \\
\hline Coronary artery disease & 24 & 40 \\
\hline Concomitant CABG & 12 & 20 \\
\hline COPD & 14 & 23.3 \\
\hline \multicolumn{3}{|l|}{ Symptom } \\
\hline Stroke & 16 & 26.7 \\
\hline TIA & 12 & 20 \\
\hline Asymptomatic & 32 & 53.3 \\
\hline \multicolumn{3}{|c|}{ Degree of right carotid stenosis (\%) } \\
\hline$<50$ & 16 & 26.7 \\
\hline $50-70$ & 15 & 23.3 \\
\hline $70-99$ & 30 & 50 \\
\hline \multicolumn{3}{|c|}{ Degree of left carotid stenosis (\%) } \\
\hline$<50$ & 14 & 23.3 \\
\hline $50-70$ & 2 & 3.4 \\
\hline $70-99$ & 44 & 73.3 \\
\hline Bilateral lesion & 14 & 23.3 \\
\hline
\end{tabular}

\begin{tabular}{|lcc|}
\hline \multicolumn{3}{|l|}{ Table 2. Operative and postoperative data of patients } \\
\hline Variable & $\mathbf{n}$ & \% of total \\
\hline Side of endarterectomy & 22 & 36.7 \\
$\quad$ Right & 38 & 63.3 \\
$\quad$ Left & & \\
Arteriotomy closure & 50 & 83.3 \\
$\quad$ Primary & 10 & 16.7 \\
Patch & 0 & 0 \\
Shunt use & & \\
Postoperative complications & 1 & 1.6 \\
Transient hemiparesis & 4 & 6.6 \\
Local hematoma & 0 & 0 \\
\hline Exploration for bleeding/hematoma & 0 & 0 \\
Myocardial infarction & 0 & 0 \\
\hline Mortality & Mean \pm SD (range) \\
\hline Variable & $20.97 \pm 7.52(7-33)$ \\
\hline Clamp duration (minutes) & $1.3 \pm 0.65(1-3)$ \\
ICU stay (days) & $3.67 \pm 1.3(3-8)$ \\
Hospital stay (days) & & \\
\hline ICU: Intensive care unit &
\end{tabular}

The present study showed a correlation of right and left sided NIRS values with each other regardless of the side of the lesion. This was true for all time points of measurements including those after clamping. Therefore to simplify the comparison of results we gave only right and left sided results. The analysis of the right and left NIRS findings in these 60 patients revealed a significant decrease between intubation and incision NIRS values ( $\mathrm{p}=0.008$ and $\mathrm{p}=0.02$ respectively). No significant change was detected in other duration times. Further analysis showed no difference between NIRS findings of right and left hemispheres at all points of recordings (Table 3 ).
Table 3. NIRS findings of right and left hemispheres at all points

\begin{tabular}{|lccc|}
\hline Measurement times & $\begin{array}{c}\text { Right sided } \\
\text { NIRS }\end{array}$ & $\begin{array}{c}\text { Left sided } \\
\text { NIRS }\end{array}$ & p \\
\hline Awake & $62.57 \pm 7.48$ & $61.80 \pm 6.77$ & 0.679 \\
Intubation & $63.20 \pm 9.96$ & $63.50 \pm 9.73$ & 0.859 \\
Incision & $59.87 \pm 8.92$ & $61.00 \pm 8.85$ & 0.623 \\
Clamp on $1 \mathrm{~min}$. & $58.33 \pm 8.86$ & $58.87 \pm 8.88$ & 0.817 \\
Clamp on $5 \mathrm{~min}$. & $59.03 \pm 8.42$ & $58.30 \pm 8.52$ & 0.739 \\
Clamp on $10 \mathrm{~min}$. & $59.25 \pm 8.79$ & $58.64 \pm 8.27$ & 0.791 \\
Clamp on $15 \mathrm{~min}$. & $60.13 \pm 7.97$ & $60.33 \pm 7.29$ & 0.925 \\
Clamp on $20 \mathrm{~min}$. & $61.31 \pm 7.69$ & $60.69 \pm 7.70$ & 0.820 \\
Clamp off $1 \mathrm{~min}$ & $60.93 \pm 8.06$ & $60.57 \pm 7.85$ & 0.859 \\
Clamp off 5 min & $60.37 \pm 8.33$ & $60.60 \pm 8.13$ & 0.913 \\
End of the operation & $60.87 \pm 8.01$ & $61.63 \pm 7.57$ & 0.705 \\
After extubation & $62.97 \pm 8.28$ & $63.37 \pm 8.18$ & 0.851 \\
\hline NIRS: Near-infrared spectroscopy & & \\
\hline
\end{tabular}

Moreover, patients with bilateral lesions showed similar pattern of NIRS changes to the patients with unilateral lesions (e.g. right and left sided NIRS in those with bilateral lesions; at incision 59.6 \pm 8.5 and $59.8 \pm 8.7$, after CC of carotid artery 58.1 \pm 8.6 and $58.5 \pm 8.7$ ).

There was no statistically significant decrease of the NIRS values of either the right or the left hemisphere after cross-clamping of the ICA and no correlation was found between blood pressure indices and the NIRS values at that time (Table 4). In three patients NIRS values dropped more than $20 \%$ from baseline values just before declamping of carotid artery. The clamp was removed from carotid artery in a very short time and a shunt was not necessary in any. No neurological deficit was observed in any of these patients.

Table 4. Correlations of NIRS values and blood pressure indices after carotid clamping

\begin{tabular}{|lccc|}
\hline & $\begin{array}{c}\text { Systolic blood } \\
\text { pressure }\end{array}$ & $\begin{array}{c}\text { Diastolic blood } \\
\text { pressure }\end{array}$ & $\begin{array}{c}\text { Mean blood } \\
\text { pressure }\end{array}$ \\
\hline \multirow{2}{*}{ Right sided NIRS } & $\mathrm{r}=0.048$ & $\mathrm{r}=0.352$ & $\mathrm{r}=0.234$ \\
& $\mathrm{p}=0.800$ & $\mathrm{p}=0.057$ & $\mathrm{p}=0.214$ \\
Left sided NIRS & $\mathrm{r}=-0.089$ & $\mathrm{r}=0.258$ & $\mathrm{r}=0.098$ \\
& $\mathrm{p}=0.638$ & $\mathrm{p}=0.169$ & $\mathrm{p}=0.606$ \\
\hline NIRS: Near-infrared spectroscopy & & \\
\hline
\end{tabular}

Almost half of our patients (53.3\%) were asymptomatic. Awake NIRS values of symptomatic and asymptomatic patients were compared to see if there was any association between symptoms and global oxygenation of cerebral hemispheres. Mean awake NIRS value on the right hemisphere was $62.71 \pm 9.7$ in symptomatic patients and was $62.4 \pm 5.1$ in asymptomatic patients $(\mathrm{p}=0.9)$ where as on the left side these values were $60.7 \pm 8.4$ in symptomatic and $62.6 \pm 4.9$ in asymptomatic cases $(\mathrm{p}=0.4)$. Therefore no association was found between NIRS values and the presence of symptoms in patients with carotid disease. 


\section{DISCUSSION}

Stroke is ranked as the third leading cause of mortality and morbidity worldwide. One-fifth of all strokes are caused by extracranial internal carotid artery stenosis (7). Carotid endarterectomy (CEA) is considered the gold standard treatment for stenosis of the internal carotid artery above $70 \%$ (6). However, despite the relatively low perioperative stroke risk of this surgical technique (0.7$1.5 \%)$, there is a high number of patients suffering from complications as 100,000 patients are operated per year in the United States only (1).

It is highly important to monitor cerebral perfusion during CEA in order to prevent neurological complications and to avoid unnecessary shunting in patients operated on under general anesthesia. Several cerebral monitoring techniques such as electroencephalogram (EEG), somatosensory evoked potentials (SSEP), Transcranial Doppler (TCD) and near-infrared spectroscopy (NIRS) are used during CEA operations (8-11).

$\mathrm{Yu}$ Wang et al. (12) found that the sensitivity and specificity of NIRS monitoring for intraoperative hypoperfusion were $64.3 \%$ and $90.0 \%$, resulting in a strong consistency with TCD monitoring results. JM Findlay et al. (13) compared stump pressures and NIRS measurements in CEA operations in terms of shunt placement and reported that NIRS was more accurate than stump pressure measurement. We routinely use NIRS monitorization during CEA operations. It is both non-invasive and offers continuous assessment of cerebral perfusion in target brain tissue $(14,15)$. We believe that one of the major advantages of NIRS monitorization is the spontaneous observation of changes in cerebral oxygenation in accordance to changes in cerebral circulation. This helps us to assess the factors changing cerebral oxygenation immediately and to react to solve any problems in a short time.

A common surgical practice is to maintain mean arterial pressure above baseline or arterial pressure above 150 $\mathrm{mmHg}$ by using vasoactive drugs during cross-clamping of the internal carotid artery (16). This is an unpublished rule of thumb and mean arterial pressure is often targeted to be approximately $20 \%$ above baseline. Although generally acceptable, this approach may not be suitable for all patients due to the undesirable outcomes such as cerebral hemorrhage and fatal cardiac events under systemic heparinization. This maneuver essentially aims to increase total cerebral blood flow as well as flow to the cerebral hemisphere ipsilateral to the clamped carotid artery by increasing collateral flow. However, our review of the literature revealed that this approach has not been justified by any study showing the clinical effects of management strategies for blood pressure during cross clamping of carotid artery. Moreover, there are no studies which evaluate the impact of keeping blood pressure close to normal on cerebral oxygenation during CEA. The analysis of the right and left NIRS findings in our patients revealed a significant decrease between intubation and incision NIRS values ( $\mathrm{p}=0.008$ and $\mathrm{p}=0.02$ respectively). The NIRS value at the time of incision was essentially picked to represent the duration while patient was asleep but carotid artery was not manipulated yet. Therefore, we interpreted the difference in NIRS values between awake state (and at the time of intubation) and at the time of incision as the impact of general anaesthesia and its effects on cerebral perfusion.

The analysis of NIRS values at other time points showed no significant difference while patient was asleep. It is important to emphasize that there was no statistically significant decrease in the NIRS values of either the right or the left hemisphere after cross-clamping of the ICA. Besides, no correlation was found between blood pressure indices and the NIRS values at that time (Table 4). The latter may be the result of blood pressure control below $150 \mathrm{mmHg}$ and therefore relatively stable blood pressure during cross clamping and after the clamp was removed. Nevertheless, the absence of reduction in NIRS values after carotid clamping suggests that CEA may safely be performed without increasing blood pressures during clamping. This is further supported by the lack of any neurological complications in this patient group. These findings are especially important for a select patient group for whom the control of blood pressure is particularly important which include those with aortic and peripheral aneurysms at risk of rupture and those at risk of cardiac events. This study is a first study to show changes in NIRS values during various stages of carotid endarterectomy and more importantly effect of blood pressure during these stages. These results may help to develop a blood pressure strategy in different patient groups.

We did not use carotid shunting in any patients in this study group. We prefer to use shunting when NIRS values drop 20-25\% from baseline and when the clamp time is anticipated to be long due to the anatomy of the patient and the carotid lesion. Although this is not our primary end point, the absence of any neurological complications and any serious decline in NIRS values suggests that carotid endarterectomy can be performed safely without shunting while keeping normal blood pressures.

We performed a subanalysis to see if there was any association between NIRS values and the presence of symptoms in patients. We couldn't find any difference in awake NIRS values of symptomatic and asymptomatic patients. This finding may imply that the symptoms are probably not related to the global cerebral oxygenation in resting state but to either instant changes in cerebral flow or embolic events. 


\section{CONCLUSION}

Considering the NIRS findings and clinical observations of the patients in the postoperative period, we think that ICA endarterectomy operations may be safe to perform in a suitable patient group while the systolic arterial blood pressure is maintained below $150 \mathrm{mmHg}$ during cross-clamping. It may thus be possible to prevent complications caused by high arterial blood pressure under systemic heparinization. Further studies with larger patient groups are needed for more accurate results.

\section{ETHICAL DECLARATIONS}

Ethics Committee Approval: The study was carried out with the permission of Ethics Committee of Okmeydan Training and Research Hospital (Ethic number: 48670771-514.10).

Informed Consent: All patients signed the free and informed consent form.

Referee Evaluation Process: Externally peer-reviewed.

Conflict of Interest Statement: The authors have no conflicts of interest to declare.

Financial Disclosure: The authors declared that this study has received no financial support.

Author Contributions: All of the authors declare that they have all participated in the design, execution, and analysis of the paper, and that they have approved the final version.

\section{REFERENCES}

1. Morris DR, Ayabe K, Inoue T, et al. Evidence-based carotid interventions for stroke prevention: state-of-the-art review. J Atheroscler Thromb 2017; 24: 373-87.

2. Lee J, Huh U, Song S, Chung SW, Sung SM, Cho HJ. Regional anesthesia with dexmedetomidine infusion: a feasible method for the awake test during carotid endarterectomy. Ann Vasc Dis 2016; 9: 295-9

3. Lam AM, Kianpour D. Monitoring for carotid endarterectomy: more or less? Anesth Analg 2015; 120: 1186-88.

4. Maillard J, Sologashvili T, Diaper J, Licker MJ, Barcelos GK. A case of persistence of normal tissue oxygenation monitored by near-infraredspectroscopy (NIRS) values despite prolonged perioperative cardiac arrest. Am J Case Rep 2019; 20: 21-5

5. Hirofumi O, Otone E, Hiroshi I, et al. The effectiveness of regional cerebral oxygen saturation monitoring using near-infrared spectroscopy in carotid endarterectomy. J Clin Neurosci 2003; 10 : 79-83.

6. Liu B, Wei W, Wang Y, Yang X, Yue S, Zhang J. Treatment strategy for bilateral severe carotid artery stenosis: one center's experience. World Neurosurg 2015; 84: 820-5.

7. Barrett KM, Brott TG. Stroke caused by extracranial disease. Circ Res 2017; 120: 496-501.

8. Li J, Shalabi A, Ji F, Meng L. Monitoring cerebral ischemia during carotid endarterectomy and stenting. J Biomed Res 2016; 31: 11-6.
9. Kragsterman B, Parsson H, Bergqvist D. Local haemodynamic changes during carotidendarterectomy-the influence on cerebral oxygenation. Eur J Vasc Endovasc Surg 2004; 27: 398-402.

10. Pennekamp CW, Immink RV, den Ruijter HM, et al. Near-infrared spectroscopy to indicateselective shunt use during carotid endarterectomy. Eur J Vasc Endovasc Surg 2013; 46: 397-403.

11. Cho JW, Jang JS. Near-infrared spectroscopy versus transcranial Doppler-based monitoring in carotid endarterectomy. Korean J Thorac Cardiovasc Surg 2017; 50: 448-52.

12. Wang $\mathrm{Y}, \mathrm{Li} \mathrm{L}$, Wang $\mathrm{T}$, et al. The efficacy of near-infrared spectroscopy monitoring in carotid endarterectomy: a prospective, single-center, observational study. Cell Transplan 2019; 28: 170-5.

13. Findlay JM, Kesarwani R, Jacka M, Marchak BE. Combined stump pressure and oximetry for shunt use during carotid endarterectomy. Can J Neurol Sci 2017; 44: 692-6.

14. David J, Zhangjie Su, Michael T, et al. Near-infrared spectroscopy in the monitoring of adult traumatic brain injury: a review. J Neurotrauma 2015; 32: 933-41.

15. Jonsson M, Lindström D, Wanhainen A, Gidlund KD, Gillgren P. Near infrared spectroscopy as a predictor for shunt requirement during carotid endarterectomy. Eur J Vasc Endovasc Surg 2017; 53: 783-91.

16. Heyer EJ, Mergeche JL, Anastasian ZH, Kim M, Mallon KA, Connolly ES. Arterial blood pressure management during carotid endarterectomy and early cognitive dysfunction. Neurosurgery 2014; 74: 245-53. 